EUROPHYSICS LETTERS

15 July 1998

Europhys. Lett., 43 (2), pp. 226-229 (1998)

\title{
Electromagnetic interference from plasmas generated in meteoroids impacts
}

\author{
Luigi Foschini $\left(^{*}\right)$ \\ Istituto FISBAT - CNR, Via Gobetti 101, I-40129 Bologna (Italy) \\ (received 3 March 1998; accepted in final form 25 May 1998) \\ PACS. 96.50Kr - Meteors and meteoroids. \\ PACS. 52.50Lp - Plasma production and heating by shock waves and compression. \\ PACS. 95.40+s - Artificial Earth Satellites.
}

\begin{abstract}
It is shown that the plasma, generated during an impact of a meteoroid with an artificial satellite, can produce electromagnetic radiation below the microwave frequency range. This interference is shown to exceed local noise sources and might disturb regular satellite operations.
\end{abstract}

Introduction. - At the end of 1998, the first modules of the International Space Station will be put in orbit around the Earth and this should open new frontiers for life in space. The intensive use of the space makes necessary to know the potential risks. The threat from meteoroids is today well known and several authors have underlined the risks connected with the impact on a spacecraft (for a review, see [1]). However, the Olympus end-of-life anomaly [2] and the recent work of McDonnell et al. [3] put a new light on these issues. The Olympus failure is a paradigmatic example: in that case, the impact with a Perseid meteoroid may have generated electrical failures, leading to a chain reaction which culminated with an early end of the mission [2]. On the other hand, McDonnell et al. [3] showed that, if the plasma charge and current production during an impact are considered, meteoroid streams can be very dangerous, even during normal conditions. It should be noted that they considered only damages by direct discharges or current injection in circuits (e.g. via the umbilical) 30.

However, there are several other ways by which the plasma could interact with the spacecraft electronics. For example, it is useful to recall the work of Cerroni and Martelli [⿶] , in which they showed that thermal forces in impact-produced plasmas could explain the magnetisation observed in the neighbourhood of lunar craters. Even if Cerroni and Martelli studied experimentally hypervelocity impacts of aluminium projectiles on basalt targets, it is possible to extend their work to general hypervelocity impacts.

Here, we show that a plasma cloud, generated during a hypervelocity impact of a meteoroid with an artificial satellite, can radiate electromagnetic energy below the microwave frequency range and, therefore, may disturb regular satellite operations.

$\left({ }^{*}\right)$ E-mail: L.Foschini@fisbat.bo.cnr.it

Typeset using EURO-TEX 
Meteoroids impacts. - It is well known that, during a hypervelocity impact, a fraction of the projectile and target materials is evaporated and even ionized [5]. A plasma cloud is then created almost instantaneously after the impact and expands into the surrounding vacuum. McDonnell et al. [3] found an empirical formula for the evaluation of charge $Q$ produced during a hypervelocity impact. This equation, rearranged in order to emphasize the projectile dimensions and density, can be written as follows:

$$
Q \simeq 3.04 \delta^{1.02} r^{3.06} V^{3.48}[\mathrm{C}]
$$

where $\delta$ is the meteoroid density $\left[\mathrm{kg} / \mathrm{m}^{3}\right], r$ its radius $[\mathrm{m}]$ and $V$ its speed $[\mathrm{km} / \mathrm{s}]$. In this paper, we consider, as a specific example, the Leonid meteoroid stream, that is the most dangerous stream known today owing to its high geocentric speed. Typical values of $\delta$ and $V$ for Leonids are, respectively, $1000 \mathrm{~kg} / \mathrm{m}^{3}$ and $71 \mathrm{~km} / \mathrm{s}$.

In order to calculate the plasma characteristic parameters (Debye length $\lambda_{D}$ and plasma frequency $\nu$ [6]), it is necessary to make further assumptions. The plasma cloud generated during an impact has been studied theoretically (e.g. [7]) and experimentally (e.g., [4], [8], [9]). A projectile in an experimental facility reaches a speed up to about $10 \mathrm{~km} / \mathrm{s}$, far below meteoroids speeds, but data obtained in such experiments allow us to make some extrapolations.

Kadono and Fujiwara [8 recently investigated the expansion of the plasma cloud: they used nylon projectiles, with a speed range between 3 and $6 \mathrm{~km} / \mathrm{s}$, impacting targets made of different materials and found that the expansion velocity of the leading edge of the plasma cloud, $u_{\max }$, is almost constant in time. The value is about twice that of the isothermal sound velocity $\left(c_{s}\right)$ :

$$
u_{\max }=\frac{2 c_{s}}{\gamma-1} \sqrt{\frac{\gamma}{3}}=\frac{2 \gamma}{\gamma-1} \sqrt{\frac{R T}{3 \mu}}
$$

where $\gamma=c_{p} / c_{v}$ is the constant ratio of specific heats, $R$ is the universal gas constant $\left[R=8.314510 \mathrm{~J} \cdot \mathrm{mol}^{-1} \mathrm{~K}^{-1}\right], T$ is the gas temperature $[\mathrm{K}]$ and $\mu$ is the mean atomic weight of the plasma cloud. For a Leonid meteoroid, we can consider an almost complete composition of carbon and a plasma temperature of $30,000 \mathrm{~K}$, taking into account that about $1 \%$ of the kinetic energy is partitioned into ionization [5]. For $\gamma$, a value of 1.7 is considered [8]. With these assumptions we obtain an expansion speed $u_{\max }=12.8 \cdot 10^{3} \mathrm{~m} / \mathrm{s}$.

Moreover, we assume that the plasma cloud is almost hemispherical during first $20 \mu$ s and thus has a radius $\rho=0.256 \mathrm{~m}$. Then, the charge $Q$ generated by an impact of a meteoroid with radius $r$, calculated with Equation (1), must be distributed in a volume about $0.035 \mathrm{~m}^{3}$. For the sake of semplicity, we have assumed a uniform distribution, even if this is not fully realistic, but it is sufficient for our purposes. Considering each atom singly ionized, we can now calculate the electron volume density $n_{e}$ and, then, the Debye length $\lambda_{D}$ and the plasma frequency $\nu$ (see Table II).

Electromagnetic interferences. - The physical meaning of the plasma characteristic parameters $\left(\lambda_{D}\right.$ and $\left.\nu\right)$ is that electrons can move, with respect to ions, a distance $\lambda_{D}$ in a time $\nu^{-1}$, before an electric field is developed in order to restore the charge neutrality. Ions and electrons can be seen as electric dipoles, with distance $\lambda_{D}$, oscillating with a frequency $\nu$. If we consider the microwave frequency limit of $\nu_{m w}=3 \cdot 10^{11} \mathrm{~Hz}$, we infer that a Leonid meteoroid with a mass up to $8 \mathrm{mg}$ is sufficient to generate a plasma cloud with a characteristic frequency lower than $\nu_{m w}$. 
TABLE I. - Examples of charge generation and plasma parameters for Leonids. For explanation of symbols, see the text.

\begin{tabular}{ccccc}
\hline$r[\mathrm{~m}]$ & $Q[\mathrm{C}]$ & $n_{e}\left[\mathrm{~m}^{-3}\right]$ & $\lambda_{D}[\mathrm{~m}]$ & $\nu[\mathrm{Hz}]$ \\
\hline $10^{-4}$ & $2.8 \cdot 10^{-3}$ & $5.0 \cdot 10^{17}$ & $1.7 \cdot 10^{-5}$ & $6.3 \cdot 10^{9}$ \\
\hline $10^{-3}$ & 3.2 & $5.7 \cdot 10^{20}$ & $5.0 \cdot 10^{-7}$ & $2.1 \cdot 10^{11}$ \\
\hline $10^{-2}$ & $3.7 \cdot 10^{3}$ & $6.6 \cdot 10^{23}$ & $1.5 \cdot 10^{-8}$ & $7.3 \cdot 10^{12}$ \\
\hline
\end{tabular}

The average power radiated by a dipole is well known from electromagnetic theory [10]. We assume that the field produced at distances $R>>\lambda_{D}$ (far field condition) by a spherical dipole distribution, with $\lambda_{D}$ as radius, is equivalent to the field of a point dipole with moment amplitude:

$$
p=\frac{4}{3} \pi e \lambda_{D}^{4} n_{e}[\mathrm{C} \cdot \mathrm{m}]
$$

where $e$ is the electron charge $[\mathrm{C}]$. For $\nu=\nu_{m w}=3 \cdot 10^{11} \mathrm{~Hz}$, we obtain $p=1.2 \cdot 10^{-23} \mathrm{C} \cdot \mathrm{m}$. Then, the average power radiated is:

$$
<P>=\frac{p^{2} \omega^{4}}{12 \pi \epsilon_{0} c^{3}} \simeq 3 \cdot 10^{-13}[\mathrm{~W}]
$$

where $\epsilon_{0}=8.854187817 \cdot 10^{-12} \mathrm{~F} / \mathrm{m}$ is the vacuum dielectric constant, $c$ is the light speed in vacuum $[\mathrm{m} / \mathrm{s}]$ and $\omega=2 \pi \nu$.

This value must be compared with the noise of satellite electronic devices. Below $6 \cdot 10^{12} \mathrm{~Hz}$, the noise has a flat power spectral density of about $4 \cdot 10^{-21} \mathrm{~W} / \mathrm{Hz}$, that is $-204 \mathrm{~dB} \mathrm{~W} / \mathrm{Hz}$ [11. If we consider a radar, which has a bandwidth of about $60 \mathrm{~dB} \mathrm{~Hz}$, and other factors that make worse, the mean noise power in a receiver is about $-146 \mathrm{~dB} \mathrm{~W}$, that is 2.5 . $10^{-15} \mathrm{~W}$. Comparing these estimates with (14), we obtain an interference of at least two order of magnitude greater than the electronic noise. It seems clear therefore that the plasma can generate an electromagnetic interference that is not negligible and can disturb the regular satellite operations. For a specific example, if we consider the International Space Station $\left(1000 \mathrm{~m}^{2}\right.$ area), exposed for 1 hour to a meteoroid flux like 1966 Leonids, there is $41 \%$ of impact probability with a meteoroid with mass equal or greater than $10^{-8} \mathrm{~kg}[12$. Such an impact flux can produce a sequence of interferences which can increase the noise level in electronic devices, then disturbing the regular satellite operations.

Conclusions. - After the Olympus end-of-life anomaly [2] and the work of McDonnell et al. [3], it seems clear that the meteoroids hazard is not restricted to a mechanical damage. Here it is suggested a new interference path, that is electromagnetic radiation emitted from the impact-produced plasma cloud. Even if the radiated power is not sufficient to destroy anything, it may disturb regular satellite operations. Further investigations should be made on specific satellite, because they require detailed information about onboard electronics, in order to calculate possible couplings and non-linearities.

Author wishes to thank Paolo Farinella, of Department of Mathematics of the University of Pisa, for constructive review. 


\section{REFERENCES}

[1] Beech M., Brown P. and Jones J., Quart. J. R. Astron. Soc., 36 (1995) 127; Foschini L.. in Atti del Secondo Convegno Nazionale di Scienze Planetarie, Bormio 1998, in press; physics/9804026, Preprint, 1998.

[2] Caswell R.D., McBride N. and Taylor A., Int. J. Impact Eng., 17 (1995) 139.

[3] McDonnell J.A.M., McBride N. and Gardner D.J., in Proceedings of the Second European Conference on Space Debris, Darmstadt 199\%, edited by KALDEICH-SCHÜRMAnn B. and HarRIS B., (ESA Publication Division, Noordwijk) 1997, pp. 391-396.

[4] Cerroni P. and Martelli G., Planet. Space Sci., 30 (1982) 395.

[5] Fechtig H., Grün E. and Kissel J., in Cosmic Dust, edited by J.A.M. McDonnell, (Wiley, Chichester) 1978, pp. 607-669.

[6] Mitchner M. and Kruger C.H., Partially Ionized Gases, (Wiley, New York) 1973.

[7] Zel'dovich Y.B. and Raizer Y.P., Physics of Shock Waves and High-Temperature Hydrodinamic Phenomena, (Academic Press, New York) 1966.

[8] Kadono T. and Fujiwara A., J. Geophys. Res. E, 101 (1996) 26097.

[9] Schultz P.H., J. Geophys. Res. E, 101 (1996) 21117.

[10] Hauser W., Introduction to the Principles of Electromagnetism, (Addison-Wesley, Reading) 1971.

[11] Kingsley S. and Quegan S., Understanding Radar Systems, (McGraw-Hill, London) 1992.

[12] Foschini L. and Cevolani G., Nuovo Cimento C, 20 (1997) 211. 